Multifunctional transition metal diboride thin films grown by magnetron sputtering with metal-ion irradiation

Babak Bakhit 


\section{Multifunctional transition metal diboride thin films grown by magnetron sputtering with metal- ion irradiation}

Babak Bakhit

\section{I.U UNGKDENG}

Thin Film Physics Division

Department of Physics, Chemistry and Biology (IFM)

Linköping University

SE-581 83 Linköping, Sweden

Linköping 2020 


\section{Preface}

This licentiate thesis is the result of research performed in Thin Film Physics Division at the Department of Physics, Chemistry and Biology (IFM), Linköping University, Sweden, between October 2016 and December 2019. The work has been focused on the growth and characterization of transition metal diboride thin films, a novel class of promising materials where crystalline phase and stoichiometry control are challenging issues.

The research acknowledges the financial support from the Swedish Research Council VR Grant 2014-5790, 2018-03957, and 642-2013-8020, the Knut and Alice Wallenberg Foundation for a Fellowship Grant and Project funding (KAW 2015.0043), the VINNOVA Grant 2018-04290, an ÅForsk Foundation grant \#16-359, and Carl Tryggers Stiftelse contracts CTS 15:219, CTS 17:166, and CTS 14:431, Swedish Government Strategic Research Area in Materials Science on Functional Materials at Linköping University (Faculty Grant SFO MatLiU No. 2009 00971), Swedish Research Council VR-RFI (\#2017-00646_9) for the Accelerator based ion-technological center and from the Swedish Foundation for Strategic Research (contract RIF14-0053) for the Tandem Accelerator Laboratory at Uppsala University. 


\section{Papers included in the thesis}

\section{Paper I.}

Controlling the $\mathrm{B} / \mathrm{Ti}$ ratio of $\mathrm{TiB}_{\mathrm{x}}$ thin films grown by high-power impulse magnetron sputtering

Babak Bakhit, ${ }^{\text {a }}$ Ivan Petrov, ${ }^{\text {a,b }}$ J.E. Greene, ${ }^{\text {a,b }}$ Lars Hultman, ${ }^{a}$ Johanna Rosén, ${ }^{\text {a }}$ and Grzegorz Greczynski ${ }^{\mathrm{a}}$

Journal of Vacuum Science \& Technology A 36, 030604 (2018)

${ }^{a}$ Thin Film Physics Division, Department of Physics (IFM), Linköping University, SE-58183

Linköping, Sweden

${ }^{\mathrm{b}}$ Frederick Seitz Materials Research Laboratory and Department Materials Science,

University of Illinois, Urbana, Illinois 61801, USA

I was involved in designing and performing the experiments. I wrote the whole draft and contributed to the final version of the manuscript.

\section{Paper II.}

Strategy for simultaneously increasing both hardness and toughness in $\mathrm{ZrB}_{2}$-rich $\mathrm{Zr}_{1}$ ${ }_{x} T a_{x} B_{y}$ thin films

Babak Bakhit, ${ }^{\text {a }}$ David L.J. Engberg, ${ }^{\text {a }}$ Jun Lu, ${ }^{\text {a }}$ Johanna Rosen, ${ }^{a}$ Hans Högberg, ${ }^{\text {a Lars }}$

Hultman, ${ }^{\mathrm{a}}$ Ivan Petrov, ${ }^{\mathrm{a}, \mathrm{b}}$ J.E. Greene, ${ }^{\mathrm{a}, \mathrm{b}}$ and Grzegorz Greczynski ${ }^{\mathrm{a}}$

Journal of Vacuum Science \& Technology A 37, 031506 (2019)

${ }^{a}$ Thin Film Physics Division, Department of Physics (IFM), Linköping University, SE-58183 Linköping, Sweden

${ }^{\mathrm{b}}$ Frederick Seitz Materials Research Laboratory and Department Materials Science,

University of Illinois, Urbana, Illinois 61801, USA

I was involved in designing and performing the experiments. I wrote the whole draft and contributed to the final version of the manuscript.

\section{Paper III.}

Self-organized columnar $\mathrm{Zr}_{0.7} \mathrm{Ta}_{0.3} \mathrm{~B}_{1.5}$ core/shell-nanostructure thin films grown by hybrid Ta-HiPIMS/ZrB2-magnetron co-sputtering

Babak Bakhit, ${ }^{1}$ Justinas Palisaitis, ${ }^{1}$ Per O.Å. Persson, ${ }^{1}$ Björn Alling, ${ }^{2}$ Johanna Rosen, ${ }^{1}$ Lars Hultman, ${ }^{1}$ Ivan Petrov, ${ }^{1,3}$ J.E. Greene, ${ }^{1,3,4}$ and Grzegorz Greczynski ${ }^{1}$

Manuscript is submitted 
${ }^{1}$ Thin Film Physics Division, Department of Physics (IFM), Linköping University, SE-58183 Linköping, Sweden

${ }^{2}$ Theoretical physics, Department of Physics (IFM), Linköping University, Linköping SE58183, Sweden

${ }^{3}$ Frederick Seitz Materials Research Laboratory and Department of Materials Science, University of Illinois, Urbana, Illinois 61801, USA

${ }^{4}$ Department of Materials Science and Engineering, National Taiwan University of Science and Technology, Taipei 10607, Taiwan

I was involved in designing and performing the experiments. I wrote the whole draft and contributed to the final version of the manuscript. 


\section{Contents}

1. Transition metal diborides.....................................................

2. Thin Film Deposition........................................................5

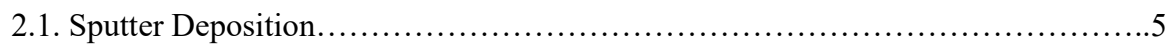

2.2. Direct Current Magnetron Sputtering......................................6

2.3. High Power Impulse Magnetron Sputtering.....................................

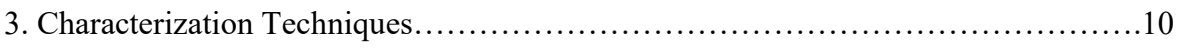

3.1. X-ray Diffraction.................................................... 10

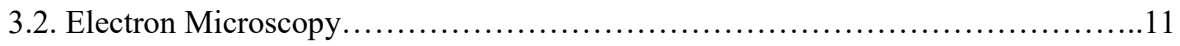

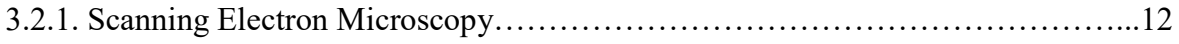

3.2.2. Transmission Electron Microscopy................................... 13

3.2.3. Analytical TEM....................................................... 15

3.2.3.1. Energy-dispersive X-ray Spectrometry................................ 15

3.2.3.2. Electron Energy-loss Spectrometry..................................... 16

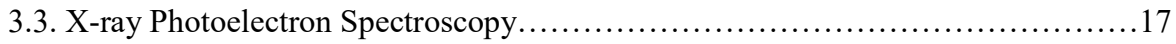

3.4. Elastic Recoil Detection Analysis..........................................18

3.5. Atom Probe Tomography................................................ 19

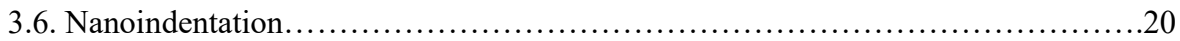

3.7. Residual Stress Measurement...................................................21

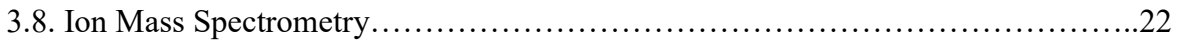





\section{Transition Metal Diborides}

Refractory transition-metal (TM) diborides, classified as ultra-high temperature materials with melting temperatures $\mathrm{T}_{\mathrm{m}} \geq 3000{ }^{\circ} \mathrm{C}$ (see Fig. 1) [1], are suitable for extreme thermal and chemical environments which include, individually or in combination, temperatures exceeding $2000{ }^{\circ} \mathrm{C}$, drastic chemical reactivity, hydrostatic pressure, mechanical stress, wear, and very high levels of heat gradients [1, 2]. Their high strength at elevated temperatures couples with good thermal conductivity, which provides a high thermal-shock resistance under severe heat fluxes, make them suitable for use in many aerospace applications such as rocket components, atmospheric reentries, jet engine turbines, and, in particular, sharp leading edges and propulsion systems in hypersonic vehicles, with speeds exceeding Mach 5 [1, 3-10]. In addition, there is also a growing demand for employing TM diborides in hightemperature electrodes [11-13], molten metal containment [1], refractory crucibles [14], thermocouple protection tubes in steel baths and aluminum reduction cells [15, 16], reinforcement fibers [17, 18], solar power [19-23], and armor applications [16]. The use of diborides in advanced nuclear fission reactors as neutron absorber materials, which are intentionally introduced into reactor cores to control the neutron balance, is also of great interest $[15,24,25]$.

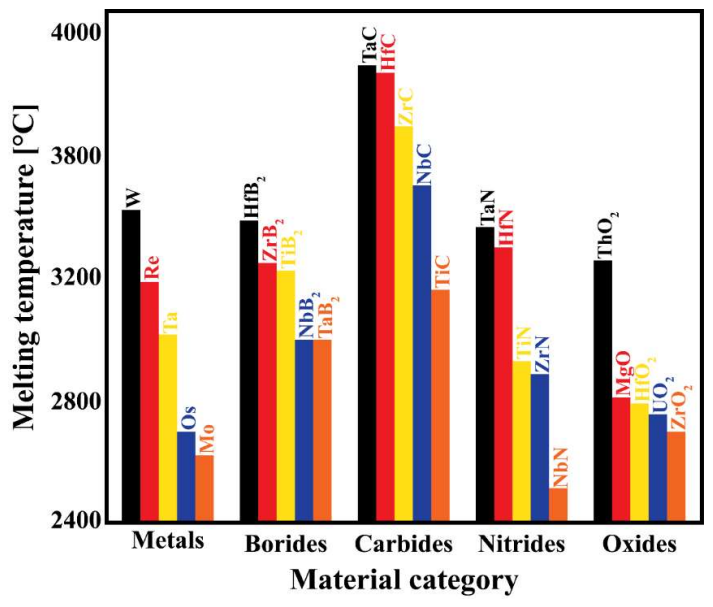

Fig. 1. Melting temperatures of some common metals, borides, carbides, nitrides, and oxides [1]. 
TM diborides have recently received increasing attention as the new class of hard ceramic thin films. They are used as wear-protective coatings on cutting tools [26-29], engine components in aerospace applications [30-34], nuclear fusion components [35-37], as well as metallic contacts in semiconductor [38] and microelectronic devices [39-41].

TM diborides typically crystallize in a hexagonal $\mathrm{AlB}_{2}$ crystal structure $(\mathrm{P} 6 / \mathrm{mmm}, \mathrm{SG}-$ 191), in which densely packed metal atom layers are held on atop positions (with respect to the neighboring metal layers) by graphite-like sheets of boron atoms [42, 43], Fig. 2. The strong covalent bonding between TM and B atoms, as well as, within the honeycomb B sheets provides high melting temperature, hardness, and stiffness [44], while metallic bonding within TM layers results in good electrical and thermal conductivities for these compounds [43]. This unique combination of ceramic and metallic properties accounts for the fact that TM diboride thin films are very promising candidates for numerous applications.

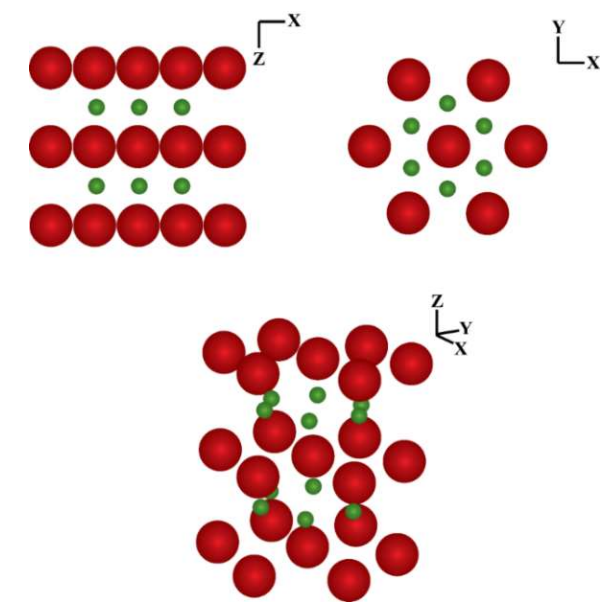

Fig. 2. Three different projections of $\mathrm{TMB}_{2}$ with the $\mathrm{AlB}_{2}$-type structure. $\mathrm{TM}$ atoms are indicated in red, while B atoms are green [45].

There are, however, challenges that need to be addressed in order to provide a wide application range for these materials. The most serious one is that TM borides, contrary to TM nitrides which have wide single-phase regions [46, 47], are line-compounds [48] for which deviations from stoichiometry, $\mathrm{B} / \mathrm{TM}=2$, can lead to the formation of secondary phases. Thus, controlling the composition of diboride layers is crucial as it has significant influence on film properties. Particularly, most common sputter-deposited TM diboride films (e.g. TM $=\mathrm{Ti}$ and 
Zr) $[49,50]$ tend to be overstoichiometric in B due to the difference in TM and B preferentialejection angles from the target coupled with mass-mismatch between the sputtering gas and target constituents affecting transport enroute to the substrate [51].

Efforts have been made to tune the composition of the sputter-deposited diboride layers. For example, increasing the sputtering pressure, and/or the target-to-substrate distance, reduces the TM deficiency due to the higher gas-phase scattering probability of light B atoms during transport to the substrate [51]. An increase in the substrate bias is also recommended; it leads to a limited decrease in the $\mathrm{B} / \mathrm{TM}$ ratio as a result of preferential $\mathrm{B}$ resputtering [49]. A successful approach for obtaining stoichiometric $\mathrm{TiB}_{2}$ thin films was recently proposed based on the use of highly-magnetically-unbalanced magnetron sputtering of a $\mathrm{TiB}_{2}$ target in $\mathrm{Ar}$ atmosphere to selectively ionize sputter-ejected $\mathrm{Ti}$ atoms, which are then steered via a tunable external magnetic field to the growing film. The $\mathrm{B} / \mathrm{Ti}$ ratio is thus controlled by varying the field strength of external Helmholtz coils [52]. However, this technique requires external magnetic coils, which is not compatible with industrial deposition systems.

The above issue is addressed in paper $\mathrm{I}$, in which it is demonstrated that the $\mathrm{B} / \mathrm{Ti}$ ratio in $\mathrm{TiB}_{\mathrm{x}}$ thin films grown by high power impulse magnetron sputtering (HiPIMS) from a $\mathrm{TiB}_{2}$ target, can be controllably tuned from 2.08 to 1.83 by adjusting HiPIMS pulse length ton between 100 and $30 \mu$ s.

Although diborides are inherently hard, this alone is not sufficient to prevent failure in applications involving high stresses, as hardness is typically accompanied by brittleness which leads to crack formation and propagation [53]. To suppress brittle cracking, films need to be both hard and relatively ductile that is referred to as high toughness, which is the resistance of a material to crack formation.

In paper II, the toughness of diborides is addressed. We propose a successful strategy to simultaneously increase both hardness and toughness of $\mathrm{ZrB}_{2}$ layers by alloying with $\mathrm{Ta}$. The hardness of the alloys grown by HiPIMS and DCMS co-sputtering (Ta-HiPIMS/ZrB2-DCMS) increases from $\sim 35.0 \mathrm{GPa}$ for $\mathrm{ZrB}_{2.4}$ to $\sim 42.0 \mathrm{GPa}$ for $\mathrm{Zr}_{0.7} \mathrm{Ta}_{0.3} \mathrm{~B}_{1.5}$, accompanied by an enhancement in indentation toughness from 4.0 to $5.2 \mathrm{MPa} \sqrt{ }$, due to a corresponding transition in nanostructure from B-rich to Ta-rich column boundaries together with a decrease in column sizes.

Investigations of the nanostructure of the $\mathrm{Zr}_{0.7} \mathrm{Ta}_{0.3} \mathrm{~B}_{1.5}$ layers on atomic scale (see paper III) reveals that these films have unique core/shell nanostructures, in which $\sim 8$ - $\AA$ cores are 
crystalline hexagonal- $\mathrm{AlB}_{2}$-structure $\mathrm{Zr}$-rich stoichiometric $\mathrm{Zr}_{1-\mathrm{x}} \mathrm{Ta}_{\mathrm{x}} \mathrm{B}_{2}$ that become increasingly Ta-rich toward outer edges. The shell structure is a narrow dense, disordered region which is Ta-rich and $\mathrm{B}$ deficient. 


\section{Thin Film Deposition}

Thin films, layers of material with the thickness in the range from nm to several $\mu \mathrm{m}[54$, 55], often possess unique properties such as high hardness, corrosion and/or wear resistance which are not attainable in bulk materials $[54,55]$. The history of man-made thin films dates back to the middle bronze age, more than 5000 years ago, when Egyptians had chemomechanically formed gold layers for decorative applications [56]. Thin films can be nowadays found almost everywhere; some examples include coatings on cutting tools, watches, eyeglasses, windows, transistors, hard drives, and solar cells. The properties of thin films are determined by their elemental composition, atomic bonding, crystalline phase content, and microstructures [57-59].

Among different deposition processes, physical vapor deposition (PVD) techniques are widespread methods for forming thin films. PVD techniques involve the creation of vapor phases (by evaporation, sputtering, cathodic arc, laser ablation, etc.) and their subsequent condensation on a substrate [60]. This chapter gives a short introduction about direct current magnetron sputtering (DCMS) and high power impulse magnetron sputtering (HiPIMS) used in this thesis; two sputter-deposition techniques which require high vacuum conditions in order to allow species to travel toward the substrate with as few collisions as possible $[55,59]$.

\subsection{Sputter Deposition}

Sputter deposition is a process of PVD thin film growth which primarily relies on plasma. A plasma is an ionized gas, containing a collection of charged particles (ions and electrons), which is electrically neutral [61]. Plasma can be artificially ignited by applying a potential through a gaseous medium between two electrodes. Since any gas usually contains a few electrons coming from natural radioactivity, these free electrons are rapidly accelerated in an electric field, generated by the voltage difference applied between the electrodes, and can collide with gas atoms (denoted here as X) [62]. In an inelastic collision, if the energy of the electron is higher than the energy required for the ionization of gas atoms, the gas atom can be ionized and release one additional electron (see equation 1). This process is called electron impact ionization [63].

$$
\mathrm{e}^{-}+\mathrm{X} \rightarrow \mathrm{X}^{+}+2 \mathrm{e}^{-}
$$


While the resulting electrons can ionize other gas atoms, the ionized atoms accelerated by the electric field bombard the cathode surface. This bombardment leads to several processes that the two most important ones are sputtering and emission of secondary electrons, which is critical for sustaining the plasma [63]. Such electrically-driven plasma is referred to as a glow discharge [64].

Any sputter deposition process, including the ones used in this thesis, relies on a phenomenon called sputtering, in which energetic incident particles colliding with the cathode material (called in this context target) generate a series of binary collisions - a collision cascade [65]. When the transferred energy is sufficiently high to overcome the binding energy at the lattice site, a primary recoil atom is produced [66]. Some of the near-surface atoms may have a favorable momentum and energy to overcome the surface binding energy and are ejected (sputtered). Sputtering efficiency is measured by sputtering yield that is the average number of sputtered atoms from a solid surface per incident ion. Sputtering yield depends on energy, mass, and angle of incident particles, as well as crystallinity, crystal orientation, surface binding energies, and mass of target atoms. For incident ions with kinetic energies below 20-40 eV, sputtering hardly occurs; however, above this threshold, the sputtering yield increases as a function of incident particle energy. After reaching a maximum, a drop in the sputtering yield takes place due to ion implantation in the target $[66,67]$.

\subsection{Direct Current Magnetron Sputtering}

For decades, DC glow discharge had been used as a sputtering source in order to deposit thin films [68]. However, this technique has a clear disadvantage which is a low sputtering rate due to a relatively low plasma density in front of the target. Since electrons travel straight into the gas and create most of ionization collisions far from the target, only a few ions return to the target. This requires high discharge voltages and high gas pressures, which in turn causes thermalization of the sputtered flux $[60,64]$.

In order to improve the efficiency of the sputtering process, the lifetime of the energetic electrons in the vicinity of the target should be increased. The common solution is to place magnets behind the target in the device called magnetron. A DC planar-magnetron sputtering configuration is schematically illustrated in Fig. 1. In this setup, the polarities of center and outer magnets are different. As a result of providing an opposite polarity, the magnetic field lines go out from the periphery of the target and go back into the center of the target. [69, 70]. 
In a conventional DC magnetron sputtering (DCMS) discharge, the electron trajectories are more complex due to the presence of both a magnetic field $\mathbf{B}$ and an electric field $\mathbf{E}$, Fig. 1.In this configuration, the electrons show a net drift perpendicular to both $\mathbf{B}$ and $\mathbf{E}$ field vectors (referred to as the Hall drift or the $\mathbf{E} \times \mathbf{B}$-drift) and perform trochoid movements. The magnetic confinement results in a torus-shaped dense plasma in front of the target which causes a nonuniform sputtering erosion, leading to the formation of an erosion pattern on the target called race track. The orbit radius of ions, on the other hand, is significantly larger which can exceed the chamber size. For example, in a deposition system with $400 \mathrm{G}(0.04 \mathrm{~T})$ magnetic field, the orbit radius of argon ions is $\sim 51 \mathrm{~cm}$ by applying a $500-\mathrm{V}$ potential to the target [61]. Hence, the effect of magnetic field on the ion trajectory can be neglected. The magnetic fields significantly confine the movement of the electrons which leads to trapping them in the target vicinity and increasing ionization probability (ionization cross section) for sputter-ejected species.

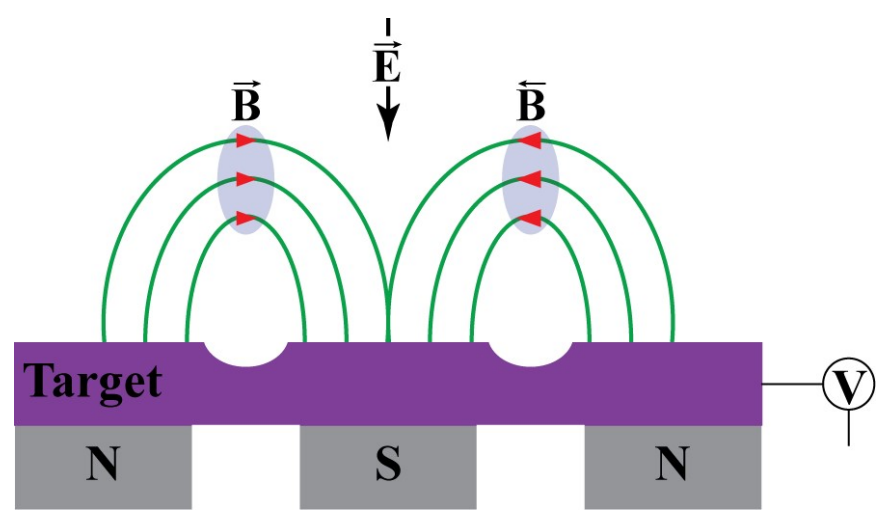

Fig. 1. A schematic of the DC planar-magnetron used for sputtering, in which all magnets have the same strength.

The primary advantages of magnetron sputtering systems are high deposition rates, low operating pressures, and the ease of industrialization [68]. The maximum deposition rate in magnetron sputtering process is, among other factors, limited by the cooling ability of target because applying high powers causes target overheating and melting.

\subsection{High Power Impulse Magnetron Sputtering}


High power impulse magnetron sputtering (HiPIMS) is an ionized PVD method which relies on the application of short (20 to $200 \mu$ s), low frequency (50 to $1 \mathrm{kHz}$ ), high-voltage (500 to $2000 \mathrm{~V}$ ) pulses to produce high density plasmas with high degrees of sputter-ejected atoms ionization [71-78]. Since the average HiPIMS powers do not exceed the levels used in conventional DCMS, the heat load to target is similar to that during DCMS [71]. The advantage is that no major modification of the existing sputtering systems is required and dedicated HiPIMS power supplies can be directly connected to the conventional magnetron-sputtering equipment. In addition, significantly fewer macro-particles are generated in a HiPIMS discharge compared to cathodic arc, which is known as a PVD process providing a highlyionized discharge [75].

Applying pulsed powers with high amplitudes in HiPIMS leads to a significant increase in electron density in front of the target surface, which depends on target material, nature and pressure of working gas [78]. The ionization mean free path, defined as an average distance gas atoms travel before getting ionized, for the conventional DCMS with an electron density of $10^{17} \mathrm{~m}^{-3}$ is around $50 \mathrm{~cm}$, while in the case of HiPIMS, with a peak electron density of $\sim 10^{19}$ $\mathrm{m}^{-3}$, decreases to approximately $1 \mathrm{~cm}[79,80]$. As a result, the high electron density in a HiPIMS discharge causes effective ionization of the sputter-ejected atoms. In addition, depending on the target material [81], self-sputtering may also take place in which the ionized sputter-ejected atoms are back-attracted toward the target causing further sputtering. This phenomenon is one of the reasons for that the power-normalized HiPIMS deposition rate is lower than for DCMS. Ionization of the sputter-ejected atoms also gives an opportunity to control their energy once they impinge on the growing film surface by applying a negative bias voltage to the substrate $[73,82-84]$.

The particular shapes of current and voltage waveforms during HiPIMS depend on many parameters such as target material, average applied power, length and frequency of pulses, gas composition and pressure, magnetron configuration, and also the size of the capacitor bank in the power supply [73, 85, 86]. Some examples are included in Fig. 2 which shows the effect of varying the HiPIMS pulse length on current waveforms for $\mathrm{a}^{\mathrm{TiB}_{2}}$ target operated at $3 \mathrm{~kW}$ and $1 \mathrm{kHz}$ in pure $\mathrm{Ar}$ at $450{ }^{\circ} \mathrm{C}$ [87]. There is an increase in peak target current from $0.27 \mathrm{~A} / \mathrm{cm}^{2}$ for $100-\mu$ s pulses to $0.88 \mathrm{~A} / \mathrm{cm}^{2} 30$ - $\mu$ s pulses. The peak target-current density is a sensitive function of the applied power, pulse length, and pulse frequency. 


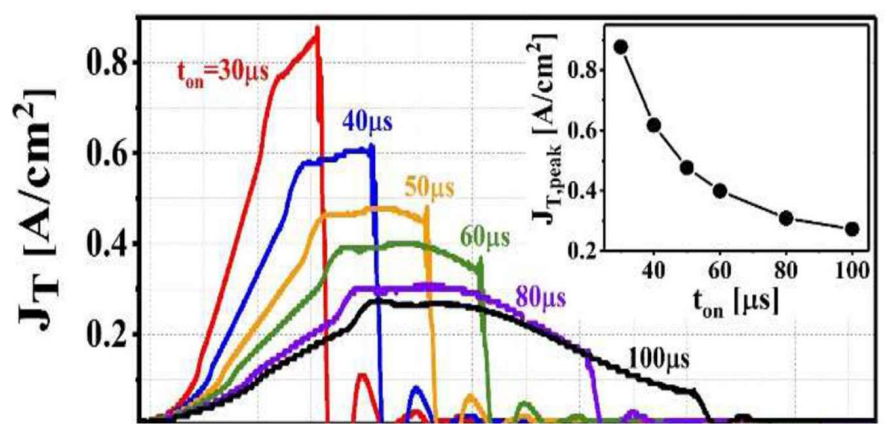

Fig. 2. Target current density waveforms recorded during HiPIMS sputtering of $\mathrm{TiB}_{2}$ in Ar at $3 \mathrm{mTorr}$ as a function of the pulse length. The target power is $3 \mathrm{~kW}$ with a $1 \mathrm{kHz}$ [87].

An additional feature of HiPIMS discharges is a time separation between metal- and gas-ion fluxes incident at the substrate due to the rarefaction of working gas in front of the target $[84,88]$. This effect can be utilized during the film growth to independently control the momentum and energy of incident ionized species through the application of substrate bias pulses (metal-ion-synchronized HiPIMS) [88-90]. This approach has been employed in papers II and III of this thesis.

A new film-growth pathways have been recently demonstrated by combining metal-ionsynchronized HiPIMS with a second magnetron operating in DCMS mode. This hybrid method provides the opportunity to tune the effect of selected energetic metal-ion fluxes on the properties of pseudobinary and higher-order TM-based alloys, revealing fundamental differences among the effects of different metal-ion fluxes, while still maintaining a high deposition rate. The use of hybrid HiPIMS/DCMS provides a powerful control over the microstructure and properties of thin films such as density, hardness, adhesion, roughness, and phase formation. More details about HiPIMS plasma processing can be found in [78, 85], while the hybrid HiPIMS/DCMS deposition is discussed in [58]. 


\section{Characterization Techniques}

This chapter gives an overview of the analytical techniques used to characterize TM diboride thin films studied in papers I-III of this thesis.

\subsection{X-ray Diffraction}

$\mathrm{X}$-ray diffraction (XRD) is considered a classical nondestructive method for acquiring information about crystal structure, phase content, preferred crystal orientations (texture), average grain size, crystallinity, strain, and crystal defects. The technique does not require any specific sample preparation. When X-ray photons, with sufficiently short wavelength -- the same order of magnitude as the interatomic spacing, elastically interact with atoms in a lattice, they are diffracted. In an elastic scattering event (Thomson scattering), the X-rays do not lose their energy, i.e. the wavelength remains the same, while the direction is changed. The in-phase scattered X-rays undergo constructive interference when Bragg's law is fulfilled [91];

$$
\mathrm{n} \lambda=2 \mathrm{~d}_{\mathrm{hkl}} \cdot \sin \theta
$$

where $\mathrm{n}$ is an integer called the order of reflection, $\lambda$ is the wavelength of X-rays, $\mathrm{d}$ is the characteristic spacing between the specimen crystal planes and $2 \theta$ is the angle between the incident and the diffracted beams. However, not all lattice planes which fulfill the Bragg's law will give rise to diffraction reflections. The allowed reflections must also obey the structure factor relationship determined by the crystal structure. The result of such a scattering interaction is a diffraction pattern in which the intensity of the scattered X-rays is plotted as a function of the scattering angle, 20. For certain angles, the scattered X-rays interfere constructively, resulting in reflections with sharp intensities. As these intense reflections appear at specific angles which correspond to specific unit cell dimensions and symmetry, the diffraction patterns are treated as a fingerprint of the crystal structure. While the diffraction angles depend on the Bravais lattice and unit cell dimensions, the diffracted intensities change with the atomic number of the constituent atoms and their geometrical relation with respect to the lattice points.

$\mathrm{X}$-rays can lose their energy during transiting through a material due to interaction with material core electrons. This inelastic energy loss, called energy absorption, follows the BeerLambert law [91];

$$
\mathrm{I}(\mathrm{x})=\mathrm{I}_{0} \cdot \mathrm{e}^{-\mu \mathrm{x}}
$$


where $\mathrm{I}(\mathrm{x})$ is the $\mathrm{X}$-ray intensity at depth $\mathrm{x} ; \mathrm{I}_{0}$ is the initial intensity; $\mu$ is the linear absorption coefficient, which depends on radiation wavelength, the composition and density of the material; and $\mathrm{x}$ is the distance that $\mathrm{X}$-rays travel inside the material. The absorption can change scattered intensities in a diffraction pattern.

The most commonly used XRD geometry is called Bragg-Brentano geometry. This is a parafocusing geometry where divergent X-rays hit the sample and are then refocused on the receiving slit before entering a detector. In this setup, the diffraction vector is always normal to the sample surface. This arrangement provides a unique combination of intensity peak shape together with angular resolution for the widest number of samples. For this thesis, two types of X-ray measurements are performed in this geometry; symmetric $\theta-2 \theta$ scans and $\omega$ scans (rocking curve). The $\theta-2 \theta$ scan gives information about diffraction peak positions, shapes, and intensities. This is a symmetrical measurement in which the sample is positioned at the center of the instrument, and both the incident and reflected angles are kept the same and change simultaneously during the measurement. Hence, due to the scattering geometry, only the crystal planes which are parallel to the sample surface give rise to diffraction reflections. In a rockingcurve measurement, which can be used to obtain the substrate curvature and thereby the residual stress, the detector is fixed to the centroid position, 20, of the Bragg reflection under investigation, while the sample is tilted, or rocked, in the vicinity of the Bragg angle. In such a scan, the $\theta$ and $2 \theta$ axes are decoupled, and the $\theta$ angle is called $\omega$. More details about XRD can be found in references [91-95].

In this thesis, $\theta-2 \theta$ XRD scans are carried out using a Philips X'Pert X-ray diffractometer with a $\mathrm{Cu} \mathrm{K}_{\alpha}$ source $(\lambda=1.5406 \AA)$ to determine crystal structure and orientation. The rocking-curve measurements are performed in a PANalytical Empyrean high-resolution Xray diffractometer. The results of the rocking-curve measurements are used in paper II to obtain the substrate curvatures required for determining films residual stresses. In both types of measurements, the diffractometers are operated at $45 \mathrm{kV}$ and $40 \mathrm{~mA}$.

\subsection{Electron Microscopy}

Electron microscopy (EM) is a common name for the range of techniques in which microstructure, morphology, and chemistry of materials can be investigated by using a beam of accelerated electrons that is focused on specimen. The fundamental advantage of EM techniques, over optical microscopy, is the possibility of achieving atomic-resolution imaging 
as the electrons have much shorter wavelengths $\lambda(\sim 2$ pm at $300 \mathrm{kV})$ than visible light (380$740 \mathrm{~nm}$ ). The resolving power of a microscope, image resolution, is expressed by the Rayleigh wave front criterion [96];

$$
\mathrm{d}=0.61 \lambda /(\mu \cdot \sin \beta)
$$

where $\lambda$ is wavelength; $\mu$ is the refractive index; and $\beta$ is the semi-angle of magnification. This equation shows the smallest observable distance between two points. The wavelength of the electrons can be varied by changing the electrons accelerating voltage $\mathrm{eV}$. There is an inverse relationship between $\lambda$ and $\mathrm{eV}$ [96];

$$
\lambda=\mathrm{h} /\left(2 \mathrm{~m}_{0} \cdot \mathrm{eV}\right)^{1 / 2}
$$

where $\mathrm{h}$ is the Plank's constant and $\mathrm{m}_{0}$ is the electron mass, which shows that the wavelengths of the electrons can be decreased, and hence resolution increased, by increasing the accelerating voltage. However, electromagnetic lenses, which are used for focusing the electron beam, suffer from aberrations (spherical and chromatic) which limit the resolution.

When an electron beam interacts with atoms of a given material, the electrons can lose their energy due to plural and multiple scattering, which respectively refer to several largeangle and many small-angle scattering events, and absorption. The volume of the material which is affected by a focused electron beam impinging the surface of a specimen has a teardrop shape and is referred to as the excitation volume. The size of the excitation volume extends from $\sim 100 \mathrm{~nm}$ to $\sim 5 \mu \mathrm{m}$ depending on the electron energy, the atomic number $(Z)$ and density of the material. While the electrons can penetrate deeper by increasing the accelerating voltage, the size of the excitation volume is more limited for materials composed of heavy elements

(high Z). Various signals can come out from different depths of the excitation volume including Auger electrons, secondary electrons (SE), back-scattered electrons (BSE), characteristic Xrays, Bremsstrahlung, and cathodoluminescence (CL). All these signals can be detected and give specific information about the material [96].

\subsubsection{Scanning Electron Microscopy}

Scanning electron microscopy (SEM) classified as an easy-to-operate electron microscopy technique relies on scanning the surface of a material by a focused beam of accelerated electrons. SEM has a spatial resolution between 1 and $10 \mathrm{~nm}$ which can be tuned 
by changing the accelerating voltage from $0.5 \mathrm{keV}$ to $30 \mathrm{keV}$. Secondary electrons (SE) and back-scattered electrons (BSE) are typically used for imaging. BSE imaging is recommended for materials containing phases with different atomic mass densities (relatively high differences). Imaging is performed by scanning the material line by line in a raster manner. The number of SE, which is the most common type of signals for SEM imaging, determines the brightness of each pixel in SEM images. SE images reveal better the topography of specimen. More details about SEM can be found in references [97].

In this thesis, the cross-sectional and plan-view morphologies of the diboride thin films are imaged using the secondary electrons. In addition, SEM is utilized to determine the average thickness of the films from the fracture cross sections.

\subsubsection{Transmission Electron Microscopy}

Transmission electron microscopy (TEM) is a powerful tool for providing specimen images with atomic resolution, electron diffraction patterns, chemical information, and chemical bonding in materials. In TEM, a parallel electron wave which is accelerated by a voltage between 100 and $300 \mathrm{keV}$ eliminates a thin specimen and penetrates through it to create the projected images or diffraction patterns of the specimen, which is then recorded. The electron mean free path $\lambda$ at this energy range varies from $\sim 37.0 \times 10^{-4} \mathrm{~nm}$ to $\sim 19.7 \times 10^{-4} \mathrm{~nm}$. Hence, TEM specimen are required to be sufficiently thin $(<\sim 100 \mathrm{~nm})$ in order to be electron transparent, which makes the specimen preparation challenging and time consuming. Since the specimen is thin, the large teardrop interaction volume is missing, and the excitation volume is close to the one determined by the electron beam spot size and the local thickness. The TEM column consists of two main parts; an illumination system and an imaging system. The illumination system has an electron gun, an accelerator and condensing lenses, while the imaging system contains objective lens, projection lenses, and detectors [96].

Two typical modes for generating image contrast in TEM are called bright-field (BF) and dark-field (DF) imaging. In the bright-field mode, which is the most common mode the unscattered (direct) electron beam is chosen by blocking the scattered electrons with the objective aperture. As a result, areas which absorb and/or scatter electrons (e.g. crystalline areas or high mass materials) appear darker, while the areas which transmit electrons look brighter. In contrast, in the dark-field mode, the scattered electrons are selected. Hence, the areas where 
electron scattering does not occur appear dark, while the areas where electrons are scattered strongly appear bright. Using this mode is recommended for studying crystal lattices, defects, stacking faults, and dislocations, with enhanced contrast. A big advantage of TEM is its ability to generate reciprocal space information, in the form of a diffraction pattern, from the imaged area providing information about crystal structure, specimen orientation, etc. [96]. The DF image with a given Bragg reflection provides a micrograph for that particular crystallographic phase. Fig. 1 compares typical plan-view BF-TEM and DF-TEM images of $\mathrm{Zr}_{0.7} \mathrm{Ta}_{0.3} \mathrm{~B}_{1.5}$ films, given in paper III. The BF-TEM image, Fig. 1(a), reveals that the film nanostructure consists of nanocolumns with a contrast difference between lighter column cores and darker shell regions, while the DF-TEM image in Fig. 1(b) exhibits stronger scattering, giving rise to higher intensities, from shell areas.

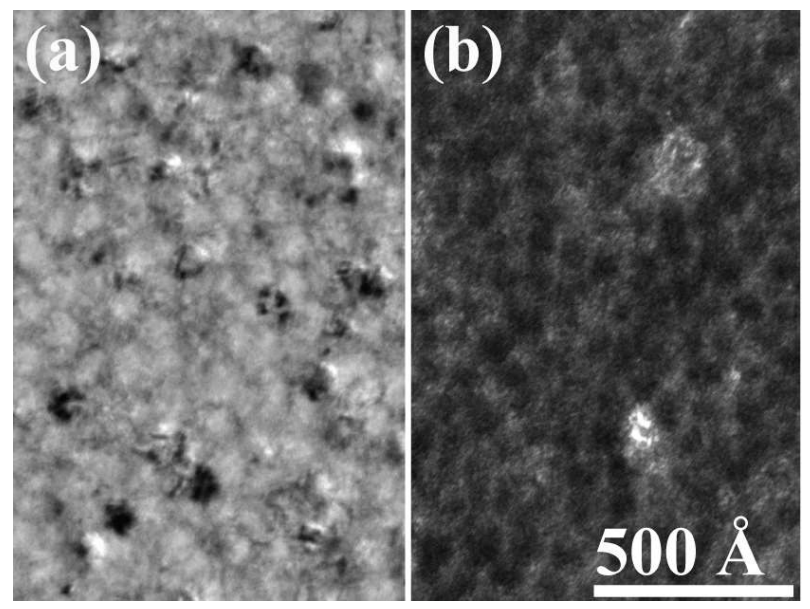

Fig. 1. Plan-view (a) bright-field TEM and (b) dark-field TEM images of $\mathrm{Zr}_{0.7} \mathrm{Ta}_{0.3} \mathrm{~B}_{1.5}$ films grown on $\mathrm{Si}(001)$ substrates at $475{ }^{\circ} \mathrm{C}$ in pure Ar at $3 \mathrm{mTorr}(0.4 \mathrm{~Pa})$ by hybrid TaHiPIMS/ZrB 2 -DCMS co-sputtering with 50- $\mu$ s HiPIMS pulses.

Compared to TEM, in which the parallel beam of electrons passes through a specimen to form an image, in a scanning TEM (STEM), the electron beam is focused to a fine spot with an spot size down to $60 \mathrm{pm}$ which is scanned over the specimen in the same fashion as in SEM. In STEM, the image contrast is based on evaluating the intensities of elastically incoherently scattered electrons by the specimen, with a relatively large scattering angle $\left(>3^{\circ}\right)$, which are 
detected by a high angle annular dark field (HAADF) detector. STEM-HAADF imaging is typically referred to as Z-contrast imaging which is sensitive to the elemental number and specimen thickness. Since the scattering angle significantly depends on the atomic number, areas with higher masses appear brighter in STEM images. STEM probes are typically combined with spectroscopic mapping for performing energy dispersive X-ray (EDX) spectroscopy and electron energy loss spectroscopy (EELS) maps [96].

The latest technological developments allow modern TEM to be equipped with monochromator, which improves the energy resolution for analytical work and aberrationcorrector which greatly improves the spatial resolution. In the current work, a monochromated and aberration-corrected FEI $\operatorname{Titan}^{3}$ TEM, operated at $300 \mathrm{kV}$, is used to obtain the microstructure of the diboride thin films from both cross-sectional and plan-view sides. In papers II and III, bright-field and dark-field images are acquired in TEM mode, while Z-contrast images are acquired in STEM-HAADF imaging mode.

\subsubsection{Analytical TEM}

\subsubsection{Energy-dispersive X-ray Spectrometry}

Energy-dispersive X-ray spectrometry (EDX) is an analytical technique which can be utilized in combination with SEM, TEM, and STEM. EDX is based on analyzing the energies of characteristic X-rays emitted due to the ionization of specimen atoms, enabling elemental identification and quantification. Strong electron-matter interaction results in excitation of the inner orbital electrons of the atom. However, the excited state is not energetically favorable, which makes the exited atom return to its ground state by transferring an electron from the outer orbital to the inner one. This process is accomplished by the release of the characteristic energy photons (X-rays) or valence electrons (Auger electron). As each element has its own characteristic X-rays appearing at specific energies, detecting such radiation is used as a chemical fingerprint for identifying the elements in the specimen. The probability for generating X-rays is higher for materials with high atomic numbers due to an increase in the electron scattering cross-section. In general, EDX is not recommended for quantifying elements lighter than sodium $(\mathrm{Z}<11)[96,97]$.

In papers II and III, STEM-EDX data are utilized for mapping $\mathrm{Zr}$ and Ta from the plan view of $\mathrm{Zr}_{1-\mathrm{x}} \mathrm{Ta}_{\mathrm{x}} \mathrm{B}_{\mathrm{y}}$ thin films. 


\subsubsection{Electron Energy-loss Spectrometry}

Electron energy-loss spectrometry (EELS) is a powerful technique for electronic (atomic bonding states) and elemental characterization in atomic scale that relies on the analysis of the energy loss distribution of the transmitted primary beam electrons once they pass through a specimen in TEM. A typical EELS spectrum, shown schematically in Fig. 2, can be split into Zero-loss, low-loss and high-loss regions. The transmitted electron beam which has zero energy-loss is referred to as the zero-loss peak (ZLP). This peak is very intense and has a width which serves as an energy resolution measure of the system (primary beam and spectrometer). While the low-loss region $(<\sim 50 \mathrm{eV})$ gives electronic information from the more weakly bound conduction and electrons in the valence band, the high-loss region $(>\sim 50 \mathrm{eV})$ contains elemental information from the more tightly bound, core-shell electrons as well as details from bonding and atomic distribution. Plasmon signals can appear in the low-loss region, which their intensities can give information about the relative thickness of the specimen. The characteristic elemental signals, called ionization edges, can have relatively low intensity compared to the background, depending on the specimen thickness and edge-excitation probability. In addition, bonding environment changes can be determined by EELS as they lead to shifts in the ionization edge onsets [96]. More details about TEM and spectroscopy can be found in references [97].

In paper II, EELS spectra are acquired from the columns and column boundaries of $\mathrm{Zr}_{1}$ ${ }_{x} \operatorname{Ta}_{x} B_{y}$ thin films, from plan-view specimen, in order to quantify $B$ concentrations.

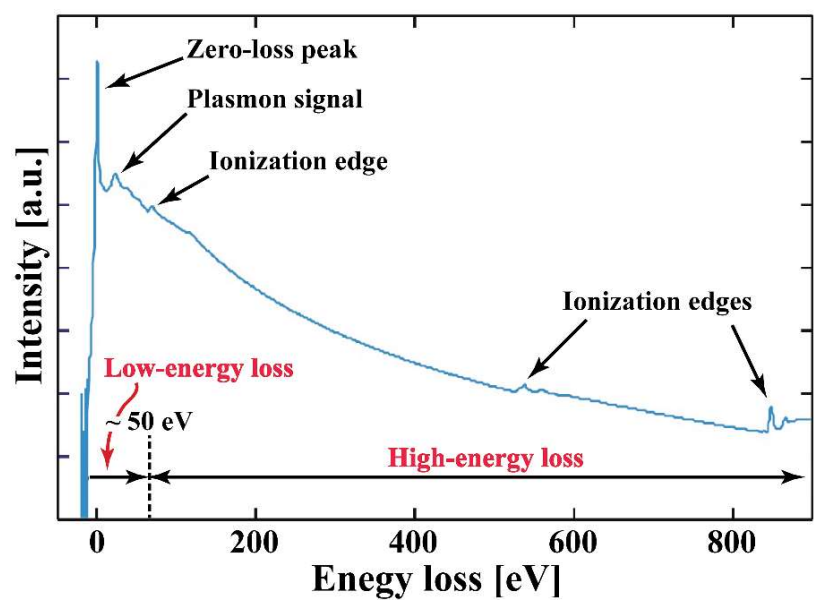

Fig. 2. A schematic illustration of an EELS spectrum plotted in logarithmic intensity scale. 


\subsection{X-ray Photoelectron Spectroscopy}

X-ray photoelectron spectroscopy (XPS) is an important surface analysis technique for determination of surface chemistry, bonding structure, and elemental composition. In XPS, an electron is ejected from a core level of a material atom by an X-ray photon with energy $\mathrm{h} v$. The kinetic energy of the emitted photoelectron $\left(E_{k}\right)$ is then measured by an electron energy analyzer. The binding energy of the photoelectron $\left(E_{b}\right)$ is obtained from the Einstein's relationship [98];

$$
\mathrm{h} v=\mathrm{E}_{\mathrm{k}}+\mathrm{E}_{\mathrm{b}}+\Phi
$$

where $\Phi$ is the spectrometer work function. In general, XPS can detect all elements with Z $>3$ (Li).

The strength of XPS is that the information about chemical environment of an atom can be obtained due to the effect referred to as the chemical shift. The charge on the atom, prior to photoemission, plays a significant role in the magnitude of the chemical shift. When an element is doped by another element, if the electronegativity of the doping element is higher than the base element, the electron density around the base element decreases due to the extra columbic interaction and the binding energy increases. However, if the electronegativity of the doping element is lower, the electron density around the base element increases and the binding energy decreases. For example, the C 1s chemical shifts is larger for C-F group than for C-O compounds as $\mathrm{F}$ is more electronegative than $\mathrm{O}[98,99]$.

The photoelectrons which leave the material without losing energy contribute to the characteristic peaks in the XPS spectra (core-levels), while those which scatter inelastically form the background on the higher-binding-energy side of the main core level peak. Apart from emission of electrons, the relaxation after the photoionization event can also proceed through the emission of an X-ray photon (X-ray fluorescence) or by the ejection of an Auger electron (Auger electron spectroscopy). Both signals may appear in the XPS spectrum. XPS spectra can be further complicated by the presence of X-ray satellites and/or X-ray ghost lines. The X-ray satellites originate from non-monochromatic radiation, while the $\mathrm{X}$-ray ghosts lines come from unsuspected X-rays irradiating the sample; for example, from misaligned or damaged anode $[98,99]$. 
Majority of samples included in this thesis was subjected to the in situ surface cleaning step prior to XPS analyses in order to remove surface oxides and contaminations. This was done by means of ion etch with energetic beam of $\mathrm{Ar}^{+}$ions rastered over an area of $3 \times 3 \mathrm{~mm}^{2}$. Such procedure is not free from artefacts which include ion implantation, atomic mixing, segregation, preferential sputtering, and an increased surface roughness. More details about XPS can be found in references [98-101].

In paper I, the elemental compositions of $\mathrm{TiB}_{\mathrm{x}}$ thin films are determined using XPS. In paper II, XPS is utilized in order to obtain information about chemical bonding in $\mathrm{Zr}_{1-\mathrm{x}} \mathrm{Ta}_{\mathrm{x}} \mathrm{B}_{\mathrm{y}}$ thin films.

\subsection{Elastic Recoil Detection Analysis}

Elastic recoil detection analysis (ERDA) is a non-destructive nuclear analytical technique which is mainly used for depth profiling and compositional characterization of thin films. In ERD measurements, a beam of positive ions strikes a solid target (sample). When enough energy is transferred from the incident ions to the target nuclei during their elastic collisions, the target atoms are recoiled from the target surface. These recoils can be then detected to determine their energies. The mass of recoil atoms is calculated assuming elastic collisions, while the depth information is determined from the energy loss of the ions and recoil atoms in the target. In conventional ERDA, unambiguously determining the recoil mass and the depth of a scattering event is difficult (referred to mass-depth ambiguity). This issue can be resolved by using a time-of-flight ERDA arrangement, schematically illustrated in Fig. 3. Determining the flight duration of a given particle, by recording start and stop signals together with the particle energy, provides a possibility to discriminate between different masses [102].

The other challenging issue is that a recoil atom cannot always be distinguished unmistakably from a scattered projectile experiencing different events (referred to recoilprojectile ambiguity). Choosing a suitable experimental geometry which does not allow the incident heavy ions to be scattered into the recoil detector can address this problem. In addition, depending on the material system, severe multiple and plural scattering events can significantly decrease the ERDA depth resolution [103]. For example, the depth resolution in $\mathrm{HfB}_{2}$ spectra acquired using a $36-\mathrm{MeV}^{127} \mathrm{I}^{8+}$ beam is really poor. However, by using heavy projectiles, both the range of analyzable elements and the total analyzed depth can be extended. For instance, a 
total analyzable depth of $>1 \mu \mathrm{m}$, with a depth resolution of $<5 \mathrm{~nm}$, can be reached by using an Au incident beam.

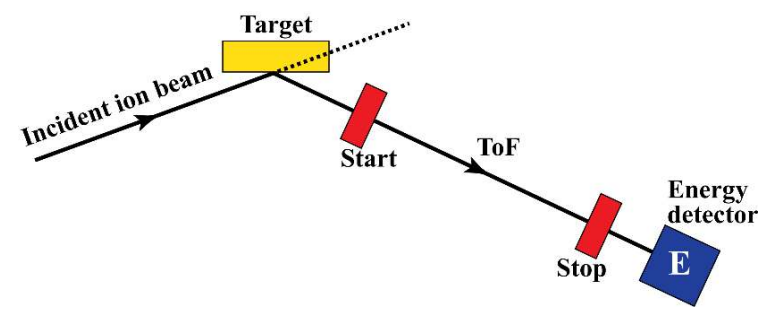

Fig. 3. A schematic illustration of ToF-E ERDA geometry [102].

ERDA is typically used for the depth-profiling of light elements in a heavy matrix. This is one of the main advantages of ERDA over other techniques like XPS and EDX, which cannot detect light elements $(Z<3)$ and provide sufficiently accurate information about their concentrations. The other advantage is that ERDA is a non-destructive technique and does not necessarily rely on surface sputter cleaning, which is essential of XPS. In addition, different isotopes of elements, $Z<13$, can be profiled and quantified by ERDA. More details about ion beam analysis techniques can be found in references [102-105].

In this thesis, ToF-E ERDA is used to obtain the elemental compositions of the thin films in all three papers.

\subsection{Atom Probe Tomography}

Atom probe tomography (APT) is a useful technique for providing 3-dimentional images of materials and measuring their chemical compositions on atomic scale, with $<1 \mathrm{~nm}$ depth and lateral resolutions. The specimen prepared in the form of a very sharp tip, with an apex diameter of $\sim 100 \mathrm{~nm}$, is biased at a high DC voltage (3-15 kV). Applying the high voltage to the tip that has a very small apex radius forms a very high electrostatic field at the tip surface which results in the evaporation of tip surface atoms. The evaporated atoms are usually ionized, which provides an ability to control and project them on a Position Sensitive Detector (PSD) with a very high detection efficiency. The tip is maintained at cryogenic temperatures during the measurement to reduce the risk of overheating which can cause surface diffusion and structural changes in the specimen. The detector simultaneously measures the time of flight and 
the 2-dimentonal position of the arrival ions. The former allows to determine the mass/charge ratio, while the latter reconstructs the original position of the atoms in the tip. Consequently, the atoms are progressively removed from the tip, and a 3-dimentional image of the material is reconstructed in atomic scale. More details about APT can be found in references [106].

In paper II, APT is used to obtain information about the nanostructure of $\mathrm{Zr}_{1-\mathrm{x}} \mathrm{Ta}_{\mathrm{x}} \mathrm{B}_{\mathrm{y}}$ thin films.

\subsection{Nanoindentation}

Nanoindentation is a technique used for measuring the mechanical responses of materials, in particular thin materials. In this technique, a diamond tip with a known geometry penetrates into the material, and the indentation load is plotted simultaneously as a function of the tip penetration depth, Fig. 4. This type of load-unload curves provides information about the elastic and plastic responses, and resistance of materials to localized plastic deformation (known as hardness). For thin films, potential substrate influence is the first necessary concern before any nanoindentation measurements. Berkovich tips are recommended for shallow indenting, due to their geometry which gives higher contact areas with the material. In addition, it is suggested to exclude indents with penetration depths $>10 \%$ of the film thickness to eliminate substrate effects. However, it has been recently shown that the effects of substrate cannot be simply eliminated by following this rule, and some experts recommend choosing indents with even lower penetration depth. Some also suggest that for indenting hard thin films, choosing hard substrates, like $\mathrm{Al}_{2} \mathrm{O}_{3}$, is better soft ones, like Si. More details about nanoindentation can be found in references [107-110].

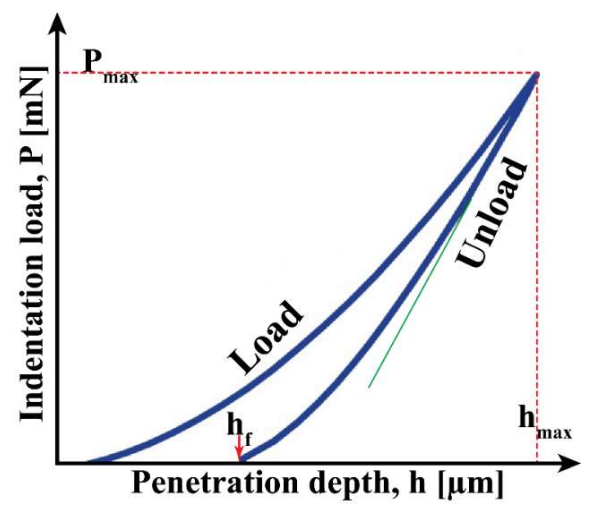


Fig. 4. A schematic illustration of indentation load vs. penetration depth curve [107].

In paper II, the hardness and elastic modulus of $\mathrm{Zr}_{1-\mathrm{x}} \mathrm{Ta}_{\mathrm{x}} \mathrm{B}_{\mathrm{y}}$ thin films are determined by nanoindentation using a Berkovich tip. In addition, the nanoindentation toughness of these layers are obtained using a diamond cube-corner tip, which is sharper and provides much higher local stresses than Berkovich tips.

\subsection{Residual Stress Measurement}

Residual stresses $\left(\sigma_{f}\right)$ have significant influences on the adhesion and properties of sputter-deposited thin films. Stresses in thin films usually comprise intrinsic stresses $\left(\sigma_{i}\right)$, which result from growth conditions and microstructural features of films and thermal stresses $\left(\sigma_{\mathrm{th}}\right)$, which result from the mismatch of thermal expansion coefficients between the substrate and films. The residual stresses of thin films are typically determined based on two different approaches by XRD; (i) $\sin ^{2} \psi$ technique, described in reference $x$, and (ii) substrate wafer curvature measurements. The $\sin ^{2} \psi$ technique is recommended for stress analysis of thin films with randomly-oriented crystallites. Films which exhibit strong preferred orientation are commonly studied by an indirect method which relies on measuring the curvature of substrate using the modified Stoney's equation [111-113];

$$
\sigma_{\mathrm{f}}=\left(\mathrm{M}_{\mathrm{s}} \cdot \mathrm{h}_{\mathrm{s}}^{2}\right) /\left(6 \mathrm{R}_{\mathrm{s}} \cdot \mathrm{h}_{\mathrm{f}}\right)
$$

where $M_{s}$ is the substrate biaxial modulus; $h_{s}$ and $h_{f}$ are film and substrate thicknesses, respectively; and $R_{s}$ is the substrate radius of curvature. Substrate curvatures can be determined before and after film deposition from rocking-curve measurements.

The thermal stresses can be calculated based on the following equation [59];

$$
\sigma_{\text {th }}=E_{f} \cdot\left(\alpha_{f}-\alpha_{s}\right) \cdot \Delta T
$$

where $E_{\mathrm{f}}$ is film elastic modulus; $\alpha_{\mathrm{f}}$ and $\alpha_{\mathrm{s}}$ are the average thermal expansion coefficients of the films and substrate respectively; and $\Delta \mathrm{T}$ is the difference between the substrate temperature during deposition and room temperature. By measuring the residual stresses $\left(\sigma_{\mathrm{f}}\right)$ and excluding the thermal stresses $\left(\sigma_{\mathrm{th}}\right)$, intrinsic stresses $\left(\sigma_{\mathrm{i}}\right)$ can be obtained. More details about residual stress in thin films together with methods for obtaining it can be found in references $[59,91$, $113,114]$. 
In paper II, the residual stresses in $\mathrm{Zr}_{1-\mathrm{x}} \mathrm{Ta}_{\mathrm{x}} \mathrm{B}_{\mathrm{y}}$ thin films are obtained using the modified Stoney's equation.

\subsection{Ion Mass Spectrometry}

Ion Mass Spectrometry is a technique for analyzing the type and the energy of ionized species present in the plasma and determination of their energy distribution functions [115]. In paper I, in-situ mass- and energy-spectroscopy analyses of ion fluxes incident at the substrate plane are performed using a Hiden Analytical EQP1000 instrument during HiPIMS sputtering of the $\mathrm{TiB}_{2}$ target. In this setup, ions enter the spectrometer via orifice which is placed at the substrate plane, facing the center of the target. Ion-energy distribution functions (IEDFs) are recorded during 100 consecutive HiPIMS pulses such that the total acquisition time per data point is $1 \mathrm{~ms}$. The ion energy is scanned between 1 and $50 \mathrm{eV}$ in $0.5 \mathrm{eV}$ steps. 


\section{References}

1. Ultra-High Temperature Ceramics : Materials for Extreme Environment Applications. 2014, [Erscheinungsort nicht ermittelbar]: John Wiley \& Sons Inc.

2. Van Wie, D.M., et al., The hypersonic environment: Required operating conditions and design challenges. Journal of Materials Science, 2004. 39(19): p. 5915-5924.

3. Padture, N.P., Advanced structural ceramics in aerospace propulsion. Nature Materials, 2016. 15: p. 804.

4. Jackson, T.A., D.R. Eklund, and A.J. Fink, High speed propulsion: Performance advantage of advanced materials. Journal of Materials Science, 2004. 39(19): p. 5905-5913.

5. Sziroczak, D. and H. Smith, A review of design issues specific to hypersonic flight vehicles. Progress in Aerospace Sciences, 2016. 84: p. 1-28.

6. Savino, R., et al., Aerothermodynamic study of UHTC-based thermal protection systems. Aerospace Science and Technology, 2005. 9(2): p. 151-160.

7. Padilla, I.D.B.a.J.F., SIMULATION OF SHARP LEADING EDGE AEROTHERMODYNAMICS, in 12th AIAA International Space Planes and Hypersonic Systems and Technologies. 15 - 19 December 2003: Norfolk, Virginia.

8. Opeka, M.M., I.G. Talmy, and J.A. Zaykoski, Oxidation-based materials selection for $2000^{\circ} \mathrm{C}+$ hypersonic aerosurfaces: Theoretical considerations and historical experience. Journal of Materials Science, 2004. 39(19): p. 5887-5904.

9. E. Wuchina, E.O., M. Opeka, W. Fahrenholtz, and I. Talmy, UHTCs: Ultra-High Temperature Ceramic Materials for Extreme Environment Applications. Electrochemical Society Interface. 16(4): p. 30-36.

10. Zhao, J.C. and J.H. Westbrook, Ultrahigh-Temperature Materials for Jet Engines. MRS Bulletin, 2003. 28(9): p. 622-630.

11. Jin, Z.J., et al., Electroforming of Copper/ZrB2 Composites Coatings and Its Performance as Electro-Discharge Machining Electrodes. Key Engineering Materials, 2005. 291-292: p. 537542.

12. Stucker, B., M. Malhotra, and X. Qu, Recent developments in selective laser sintering of ZyrkonTM EDM electrodes. International Congress on Applications of Lasers \& Electro-Optics, 1999. 1999(1): p. F158-F161.

13. B. Stucker, W.B., P. T. Eubank, S. Norasetthekul, and B. Bozkurt,, Zirconium Diboride/Copper EDM Electrodes From Selective Laser Sintering. Solid Freeform Fabric. Symp. Proc., 1997. 1: p. 257-65

14. Chapin, E. and W. Friske, A Metallurgical Evaluation of Refractory Compounds for Containing Molten Titanium. Part III-Borides and Sulfides. 1955, NAVAL RESEARCH LAB WASHINGTON DC.

15. Matkovich, V.I., Boron and refractory borides. 1977, Berlin: Springer-Verlag.

16. Basu, B. and K. Balani, Advanced structural ceramics. 2011: John Wiley \& Sons.

17. V.M. Vedula, U.-H.T.C.-C.C., Final Report on Project WRDC-TR-89-4089, Air Force Materials Laboratory, Wright Patterson Air Force Base, Dayton, OH, October 1989.

18. E.L. Courtright, H.C.G., A.P. Katz, R.J. Kerans, Ultra-High Temperature Assessment StudyCeramic Matrix Composites, Final Report WL-TR-91-4061,Wright Laboratory Materials Directorate, Wright Patterson Air Force Base, Dayton, OH, September 1992.

19. Mercatelli, L., et al., Ultra-refractory Diboride Ceramics for Solar Plant Receivers. Energy Procedia, 2014. 49: p. 468-477.

20. Sani, E., et al., Titanium diboride ceramics for solar thermal absorbers. Solar Energy Materials and Solar Cells, 2017. 169: p. 313-319.

21. Sani, E., et al., Lanthanum hexaboride for solar energy applications. Scientific Reports, 2017. 7(1): p. 718.

22. Sani, E., et al., Optical properties of boride ultrahigh-temperature ceramics for solar thermal absorbers. Journal of Photonics for Energy, 2014. 4(1): p. 1-9, 9. 
23. Sani, E., et al., Ultra-High Temperature Ceramics for solar receivers: spectral and hightemperature emittance characterization. 2012. Vol. 7. 2012.

24. Mahagin, D.E. and R.E. Dahl, Nuclear Applications of Boron and the Borides. 1977: p. 613-632.

25. Subramanian, C., A. Suri, and T. Murthy, Development of Boron-based materials for nuclear applications. Barc Newsletter, 2010. 313: p. 14.

26.

https://www.kennametal.com/en/products/20478624/556249/3925999/4283287/100 002780/grades.html.html (Kennametal Inc., Latrobe, PA); http://www.hardcoatingtech.com/documents/TiB2Sheet.pdf (Krosera Hardcoating Technologies, Monroe Falls, OH); ttp://www.harveytool.com/cms/News Archive20080927 TiB2COATING_147.aspx (Harvey Tools, Rowley, MA); http://www.maford.com/pdf/MAFord 2016 Full\%20Cat Coatings.pdf (M.A Ford, Davenport, IA).

27. Murata, Y., U.S. patent 3,487,594. (6 January 1970).

28. R. Telle, L.S.S., and K. Takagi, "Boride-based hard materials," in Handbook of Ceramic Hard Materials, edited by R. Riedel (WILEY-VCH Verlag GmbH, D-69469 Weinheim, Germany, 2008), pp. 802-945.

29. Zhou, M., et al., New Cr-B hard coatings by r.f.-plasma assisted magnetron sputtering method. Thin Solid Films, 1999. 343-344: p. 234-237.

30. Fahrenholtz, W.G. and G.E. Hilmas, Ultra-high temperature ceramics: Materials for extreme environments. Scripta Materialia, 2017. 129: p. 94-99.

31. L. A. Friedrich, N.T.R.S., 19810022661 (1981).

32. J. D. Kellner, W.J.C., and L. A. Shepard, Titanium Diboride Electrodeposited Coatings, Defense Technical Information Center, ADA047956 (1997).

33. Tului, M., G. Marino, and T. Valente, Plasma spray deposition of ultra high temperature ceramics. Surface and Coatings Technology, 2006. 201(5): p. 2103-2108.

34. Yang, X., et al., ZrB2/SiC as a protective coating for C/SiC composites: Effect of high temperature oxidation on mechanical properties and anti-ablation property. Composites Part B: Engineering, 2013. 45(1): p. 1391-1396.

35. Doyle, B.L., et al., Saturation and isotopic replacement of deuterium in low-z materials. Journal of Nuclear Materials, 1980. 93-94: p. 551-557.

36. Shikama, T., et al., Deuterium retention in Ti1-xBx films deposited onto molybdenum by cosputtering method. Journal of Nuclear Materials, 1986. 141-143: p. 156-159.

37. Windsor, C., et al., Design of cemented tungsten carbide and boride-containing shields for a fusion power plant. Nuclear Fusion, 2018. 58(7): p. 076014.

38. Nicolet, M.A., Diffusion barriers in thin films. Thin Solid Films, 1978. 52(3): p. 415-443.

39. Guziewicz, M., et al., Characteristics of sputter-deposited TiN, ZrB2 and W2B diffusion barriers for advanced metallizations to GaAs. Solid-State Electronics, 1999. 43(6): p. 1055-1061.

40. Sade, G. and J. Pelleg, Co-sputtered TiB2 as a diffusion barrier for advanced microelectronics with Cu metallization. Applied Surface Science, 1995. 91(1): p. 263-268.

41. Sung, J., et al., Remote-plasma chemical vapor deposition of conformal ZrB2 films at low temperature: A promising diffusion barrier for ultralarge scale integrated electronics. Journal of Applied Physics, 2002. 91(6): p. 3904-3911.

42. JCPDS International Centre for Diffraction Data, Z.d.Z.c.-.-.

43. Bansal, N.P., Handbook of ceramic composites. 2005, Boston MA: Kluwer Academic Publishers.

44. Bsenko, L. and T. Lundström, The high-temperature hardness of ZrB2 and HfB2. Journal of the Less Common Metals, 1974. 34(2): p. 273-278.

45. Fahrenholtz, W.G., et al., Refractory Diborides of Zirconium and Hafnium. Journal of the American Ceramic Society, 2007. 90(5): p. 1347-1364.

46. J. E. Sundgren, B.O.J., A. Rockett, S. A. Barnett, and J. E. Greene, "TiN: A review of the present understanding of the atomic and electronic structure and recent results on the growth and physical properties of epitaxial TiNx $(0.6<x<.12)$ layers," in Physics and Chemistry of 
Protective Coatings, Series 149, edited by W. D. Sproul, J. E. Greene, and J. A. Thornton (American Institute of Physics, New York, 1986), p. 95.

47. L. E. Toth, T.M.C.a.N., 1st ed. (Academic, San Diego, CA, 1971), pp. 69-101.

48. T. Lundström, T.m.b., " in Boron and Refractory Borides, edited by V. I. Matkovich (SpringerVerlag, Heidelberg, Germany, 1977), pp. 351-376.

49. Nedfors, N., et al., Influence of pulse frequency and bias on microstructure and mechanical properties of TiB2 coatings deposited by high power impulse magnetron sputtering. Surface and Coatings Technology, 2016. 304: p. 203-210.

50. Bakhit, B., et al., Strategy for simultaneously increasing both hardness and toughness in ZrB2rich Zr1-xTaxBy thin films. Journal of Vacuum Science \& Technology A, 2019. 37(3): p. 031506.

51. Neidhardt, J., et al., Experiment and simulation of the compositional evolution of Ti-B thin films deposited by sputtering of a compound target. Journal of Applied Physics, 2008. 104(6): p. 063304.

52. Petrov, I., et al., Controlling the boron-to-titanium ratio in magnetron-sputter-deposited TiBx thin films. Journal of Vacuum Science \& Technology A, 2017. 35(5): p. 050601.

53. Clegg, W.J., Controlling Cracks in Ceramics. Science, 1999. 286(5442): p. 1097.

54. Nix, W.D., Mechanical properties of thin films. Metallurgical Transactions A, 1989. 20(11): p. 2217.

55. Wasa, K., M. Kitabatake, and H. Adachi, Thin film materials technology: sputtering of control compound materials. 2004: Springer Science \& Business Media.

56. Greene, J.E., Review Article: Tracing the recorded history of thin-film sputter deposition: From the 1800s to 2017. Journal of Vacuum Science \& Technology A, 2017. 35(5): p. $05 C 204$.

57. Mayrhofer, P.H., et al., Self-organized nanocolumnar structure in superhard TiB2 thin films. Applied Physics Letters, 2005. 86(13): p. 131909.

58. Greczynski, G., et al., Paradigm shift in thin-film growth by magnetron sputtering: From gasion to metal-ion irradiation of the growing film. Journal of Vacuum Science \& Technology A, 2019. 37(6): p. 060801.

59. Ohring, M., Materials science of thin films. 2001: Elsevier.

60. Bunshah, R. and C. Weissmantel, Handbook of hard coatings. 2000.

61. Lieberman, M., "Plasma" and "Sheaths"---The Discharge Science of Irving Langmuir. Bulletin of the American Physical Society, 2005.

62. Harsha, K.S., Principles of vapor deposition of thin films. 2005: Elsevier.

63. Chen, F.F., Introduction to plasma physics and controlled fusion. Vol. 1. 1984: Springer.

64. Martin, P.M., Handbook of deposition technologies for films and coatings: science, applications and technology. 2009: William Andrew.

65. Mattox, D.M., Particle bombardment effects on thin-film deposition: A review. Journal of Vacuum Science \& Technology A, 1989. 7(3): p. 1105-1114.

66. Behrisch, R. and W. Eckstein, Sputtering by particle bombardment: experiments and computer calculations from threshold to MeV energies. Vol. 110. 2007: Springer Science \& Business Media.

67. Tracton, A.A., Coatings technology handbook. 2005: CRC press.

68. Pethrick, R.A., Advanced surface coatings Edited by D. S. Rickerby and A. Matthews Blackie \& Son Ltd, Glasgow, 1991. pp. 368, price f65.00. ISBN 0-21 6-92899-0. Polymer International, 1992. 27(2): p. 208-208.

69. Bräuer, G., et al., Magnetron sputtering - Milestones of 30 years. Vacuum, 2010. 84(12): p. 1354-1359.

70. Kelly, P.J. and R.D. Arnell, Magnetron sputtering: a review of recent developments and applications. Vacuum, 2000. 56(3): p. 159-172.

71. Helmersson, U., et al., lonized physical vapor deposition (IPVD): A review of technology and applications. Thin Solid Films, 2006. 513(1): p. 1-24. 
72. Sarakinos, K., J. Alami, and S. Konstantinidis, High power pulsed magnetron sputtering: $A$ review on scientific and engineering state of the art. Surface and Coatings Technology, 2010. 204(11): p. 1661-1684.

73. Gudmundsson, J.T., et al., High power impulse magnetron sputtering discharge. Journal of Vacuum Science \& Technology A, 2012. 30(3): p. 030801.

74. Anders, A., High power impulse magnetron sputtering and related discharges: Scalable plasma sources for plasma-based ion implantation and deposition. Surface and Coatings Technology, 2010. 204(18): p. 2864-2868.

75. Anders, A., A review comparing cathodic arcs and high power impulse magnetron sputtering (HiPIMS). Surface and Coatings Technology, 2014. 257: p. 308-325.

76. Britun, N., et al., Plasma diagnostics for understanding the plasma-surface interaction in HiPIMS discharges: a review. Journal of Physics D: Applied Physics, 2014. 47(22): p. 224001.

77. Kouznetsov, V., et al., A novel pulsed magnetron sputter technique utilizing very high target power densities. Surface and Coatings Technology, 1999. 122(2): p. 290-293.

78. Gudmundsson, D.L.T.M.J.T., High Power Impulse Magnetron Sputtering. 2019: Elsevier.

79. Bohlmark, J., et al., Spatial electron density distribution in a high-power pulsed magnetron discharge. IEEE Transactions on Plasma Science, 2005. 33(2): p. 346-347.

80. Gudmundsson, J.T., The high power impulse magnetron sputtering discharge as an ionized physical vapor deposition tool. Vacuum, 2010. 84(12): p. 1360-1364.

81. Anders, A., Discharge physics of high power impulse magnetron sputtering. Surface and Coatings Technology, 2011. 205: p. S1-S9.

82. Vlček, J. and K. Burcalová, A phenomenological equilibrium model applicable to high-power pulsed magnetron sputtering. Plasma Sources Science and Technology, 2010. 19(6): p. 065010.

83. Brenning, N., et al., A unified treatment of self-sputtering, process gas recycling, and runaway for high power impulse sputtering magnetrons. Plasma Sources Science and Technology, 2017. 26(12): p. 125003.

84. Macák, K., et al., lonized sputter deposition using an extremely high plasma density pulsed magnetron discharge. Journal of Vacuum Science \& Technology A, 2000. 18(4): p. 1533-1537.

85. Anders, A., Tutorial: Reactive high power impulse magnetron sputtering (R-HiPIMS). Journal of Applied Physics, 2017. 121(17): p. 171101.

86. Anders, A., J. Andersson, and A. Ehiasarian, High power impulse magnetron sputtering: Current-voltage-time characteristics indicate the onset of sustained self-sputtering. Journal of Applied Physics, 2007. 102(11): p. 113303.

87. Bakhit, B., et al., Controlling the B/Ti ratio of TiBx thin films grown by high-power impulse magnetron sputtering. Journal of Vacuum Science \& Technology A, 2018. 36(3): p. 030604.

88. Greczynski, G., et al., Metal versus rare-gas ion irradiation during Ti1-xAlxN film growth by hybrid high power pulsed magnetron/dc magnetron co-sputtering using synchronized pulsed substrate bias. Journal of Vacuum Science \& Technology A, 2012. 30(6): p. 061504.

89. Greczynski, G., et al., Gas rarefaction effects during high power pulsed magnetron sputtering of groups IVb and VIb transition metals in Ar. Journal of Vacuum Science \& Technology A, 2017. 35(6): p. 060601.

90. Greczynski, G., et al., Time evolution of ion fluxes incident at the substrate plane during reactive high-power impulse magnetron sputtering of groups IVb and VIb transition metals in Ar/N2. Journal of Vacuum Science \& Technology A, 2018. 36(2): p. 020602.

91. Birkholz, M., Thin Film Analysis by X-Ray Scattering. 2006: Wiley.

92. Cullity, B.D., Elements of X Ray Diffraction - Scholar's Choice Edition. 2015: Scholar's Choice.

93. Woolfson, M.M., Introduction X-Ray Crystallography. 1970: Cambridge University Press.

94. Giacovazzo, C., et al., Fundamentals of Crystallography. 2002: Oxford University Press.

95. Shih, K., X-Ray Diffraction: Structure, Principles and Applications. 2013: Nova Science Publishers, Incorporated.

96. CARTER, B.A., et al., Transmission Electron Microscopy: A Textbook for Materials Science. Diffraction. II. 1996: Springer. 
97. Brandon, D. and W.D. Kaplan, Microstructural Characterization of Materials. 2008: Wiley.

98. van der Heide, P., X-ray Photoelectron Spectroscopy: An introduction to Principles and Practices. 2011: Wiley.

99. Watts, J.F. and J. Wolstenholme, An introduction to surface analysis by XPS and AES. 2003.

100. Moulder, J.F. and J. Chastain, Handbook of X-ray Photoelectron Spectroscopy: A Reference Book of Standard Spectra for Identification and Interpretation of XPS Data. 1992: Physical Electronics Division, Perkin-Elmer Corporation.

101. Briggs, D. and M.P. Seah, Practical Surface Analysis, Auger and X-ray Photoelectron Spectroscopy. 1990: Wiley.

102. Nastasi, M., J.W. Mayer, and Y. Wang, Ion Beam Analysis: Fundamentals and Applications. 2014: Taylor \& Francis.

103. Tesmer, J.R. and M.A. Nastasi, Handbook of Modern Ion Beam Materials Analysis. 1995: Materials Research Society.

104. Nastasi, M., et al., Ion-Solid Interactions: Fundamentals and Applications. 1996: Cambridge University Press.

105. Jeynes, C., R.P. Webb, and A. Lohstroh, Ion Beam Analysis: A Century of Exploiting the Electronic and Nuclear Structure of the Atom for Materials Characterisation. Reviews of Accelerator Science and Technology, 2011. 04(01): p. 41-82.

106. Gault, B., et al., Atom Probe Microscopy. 2012: Springer New York.

107. Fischer-Cripps, A.C., Nanoindentation. 2002: Springer New York.

108. Pharr, G.M. and W.C. Oliver, Measurement of Thin Film Mechanical Properties Using Nanoindentation. MRS Bulletin, 1992. 17(7): p. 28-33.

109. Oliver, W.C. and G.M. Pharr, An improved technique for determining hardness and elastic modulus using load and displacement sensing indentation experiments. Journal of materials research, 1992. 7(6): p. 1564-1583.

110. Broitman, E., Indentation hardness measurements at macro-, micro-, and nanoscale: a critical overview. Tribology Letters, 2017. 65(1): p. 23.

111. Stoney, G.G. and C.A. Parsons, The tension of metallic films deposited by electrolysis. Proceedings of the Royal Society of London. Series A, Containing Papers of a Mathematical and Physical Character, 1909. 82(553): p. 172-175.

112. Janssen, G.C.A.M., et al., Celebrating the 100th anniversary of the Stoney equation for film stress: Developments from polycrystalline steel strips to single crystal silicon wafers. Thin Solid Films, 2009. 517(6): p. 1858-1867.

113. Carneiro, J.O., V. Teixeira, and S. Azevedo, Residual Stresses in Thin Films Evaluated by Different Experimental Techniques, in Encyclopedia of Thermal Stresses, R.B. Hetnarski, Editor. 2014, Springer Netherlands: Dordrecht. p. 4222-4231.

114. Noyan, I.C. and J.B. Cohen, Residual Stress: Measurement by Diffraction and Interpretation. 2012: Springer New York.

115. Gross, J.H., Mass spectrometry: a textbook. 2006: Springer Science \& Business Media. 



\section{Papers}

The papers associated with this thesis have been removed for copyright reasons. For more details about these see:

http://urn.kb.se/resolve?urn=urn:nbn:se:liu:diva-164911 


\section{FACULTY OF SCIENCE AND ENGINEERING}

Linköping Studies in Science and Technology, Licentiate Thesis No. 1874, 2020 Department of Physics, Chemistry, and Biology (IFM)

Linköping University

SE-58183 Linköping, Sweden

www.liu.se 